\title{
On the concept of imaging nanoscale vector fields
}

To the Editor - Lee et al. recently published the paper "Vector field microscopic imaging of light" showing intriguing maps of vectors on the nanoscale ${ }^{1}$. It claims the first experimental mapping of vector fields of light on the nanoscale. Here we argue that the general vector fields of light are not measured, as the relative phase information between field components is lost. Moreover we assert that the measured polarization signal is strongly influenced by the exact tip shape.

The most general polarization state at a random point $(x, y)$ in space is such that the endpoint of the field vector traces out an ellipse of which the axes, $\eta$ and $\zeta$, are in general not along the axes $e_{x}$ and $e_{y}$ (ref. 2). Any point $\left(E_{x}, E_{y}\right)$ on this ellipse is given by the real part of:

$$
\begin{aligned}
& E_{x}=a_{1} \exp i\left(k_{x} x-\omega t+\delta_{1}\right) \\
& E_{y}=a_{2} \exp i\left(k_{y} y-\omega t+\delta_{2}\right)
\end{aligned}
$$

where $k_{x}$ and $k_{y}$ are the $x$ and $y$ components of the wave vector, respectively, $\omega$ is the angular frequency, $t$ is time and $a_{1}, a_{2}>0$. The ellipse is inscribed in a rectangle with sides parallel to the coordinate axes and the phase difference $\delta=\delta_{1}-\delta_{2}$ determines where the ellipse touches the bounding box (Fig. 1a). Placing a polarizer in front of a detector such that only the $E_{x}$ or $E_{y}$ component of the field can reach the detector, as done by Lee et al., yields intensity signals corresponding to $E_{x} E_{x}{ }^{*}=a_{1}^{2}$ or $E_{y} E_{y}^{*}=a_{2}^{2}$. The measurement for a single setting of the analyser therefore yields the corners of the bounding box and not a point on the ellipse. Subsequent rotation of the analyser maps out how the corner of the bounding box changes as is well known for intensity-based polarization mapping. It is crucial to realize that the vectors plotted by Lee et al. are simply the long axis of such polar plots. In the limiting case of linearly (or strongly elliptically) polarized light, indeed, the direction is assigned correctly as can be seen from Fig. 1b. However in the case of circularly polarized light, as depicted in Fig. 1c, one sees that no direction for the vector can be assigned. Moreover, the length of the field vector depicted by plotting the long axis of the polar plot would be too large. This is in sharp contrast to incoherent singlemolecule field studies, which, by measuring $\left|\mathbf{p} \cdot \mathbf{E}_{\text {local }}\right|^{2}$ for different orientations of $\mathbf{p}$ (where $\mathbf{p}$ is the molecule's absorption dipole moment, and $\mathbf{E}_{\text {local }}$ is the local field), do correctly map out the local field in this case, as shown by various groups ${ }^{3-6}$. Furthermore, depicting all the obtained maxima as linearly polarized field vectors

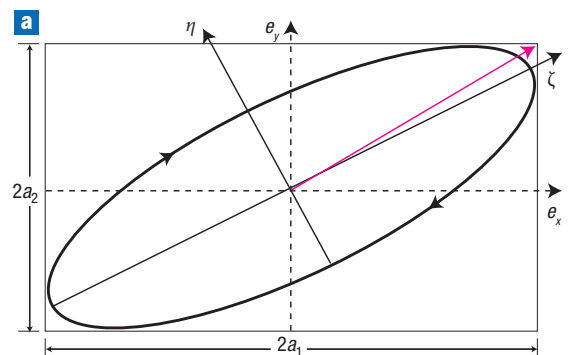

interpret, as it depends on the exact details of the probe to which field-components it is sensitive. With respect to this, it must be realized that in the experiments presented by Lee et al. different types of probes have been used.

The grounds for this concern are in particular highlighted by Fig. $3 c$ of ref. 1, in which they measure light coming through a metal slit. As a result of the electromagnetic boundary conditions, the light-vectors should, by definition, be oriented perpendicularly in close proximity to the metal ${ }^{11}$. However, close to the slit in Fig. 3 (ref. 1) the field vectors are oriented almost parallel to the metal. Comparison with the simulated result in Fig. 3d (ref. 1) indicates that the error in the orientation of the vectors close to the slit is roughly $50^{\circ}-60^{\circ}$. This is clearly not a small deviation and most probably results from a tip that has been damaged in the experiment and, as such, has become ill-defined. Note that the discrepancies between Fig. $3 \mathrm{c}$ and d remain significant in the whole near-field region until approximately $500 \mathrm{~nm}$ above the metal. This provides a clear indication that in each individual measurement, how the shape of the probe influences the observations needs to be carefully considered.

fact to measure the general field vector the retrieval of $\delta$ is essential. Measurement of this phase difference is possible by using interferometric methods in which one measures the interference between the scattered light and a known reference. In fact, such approaches have been used by several groups to demonstrate direct retrieval of the polarization, amplitude and phase of light fields on the nanoscale ${ }^{7-9}$.

Next we want to emphasize that the measured response depends strongly on the exact details of the tip used and how it interacts with the sample, as the whole method relies on the polarizability of the probe being used. Lee et al. model the tip as an effective dipole driven by the field in the absence of the probe and refer to one of our papers ${ }^{10}$ for the model.

This is a strange choice, especially as this paper points out that the scattered field depends heavily on the type and material of the tip. An all-metal or all-dielectric tip is more sensitive to longitudinal fields, whereas particles are sensitive to the total fields. Furthermore, depending on the material, a contrast reversal takes place so that for dielectric probes and particles the scattering would actually be reduced. This all indicates that the measurements shown by Lee et al. for the case where the probe is not well defined are extremely difficult to

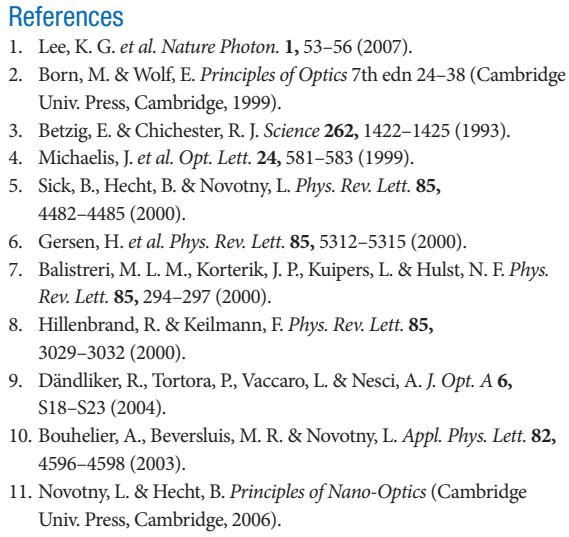

H. Gersen ${ }^{1 *}$, L. Novotny ${ }^{2}$, L. Kuipers ${ }^{3}$ and N. F. van Hulst ${ }^{4}$

${ }^{1}$ Nanophysics and Soft Matter Group,

Department of Physics, University of Bristol,

BS8 1TL, UK

${ }^{2}$ The Institute of Optics, University of Rochester,

Rochester, New York 14627, USA

${ }^{3}$ Center for Nanophotonics, FOM Institute

for Atomic and Molecular Physics (AMOLF),

Kruislaan 407, 1098 SJ Amsterdam,

The Netherlands

${ }^{4} \mathrm{ICFO}$ (Institut de Ciències Fotòniques),

Mediterranean Technology Park, 08860

Castelldefels, Barcelona, Spain

*e-mail:h.gersen@bristol.ac.uk 